\title{
Research and Implementation of ROHC for PoC Service Based on TD-SCDMA Network
}

\author{
Li Hai-wen, Huang Wei, Li Li-fu,Yu Gang \\ Chongqing Communication Institute, Chongqing China
}

\begin{abstract}
PoC is a half-duplex PTT service using VoIP technology based on cellular network packet domain carrying. ROHC is used to implement the header compression in PS domain, which can improve the transmission efficiency of the air interface. This paper describes the working principle and the modes of operation about ROHC, designs the realization processing of the compressor and decompressor. Simultaneity an improved algorithm applied to the $U$ mode is brought forward against PoC service features, and can improve the robustness of $\mathrm{ROHC}$ and the QoS of PoC session.
\end{abstract}

Keywords: PoC; VoIP; ROHC; compressor; decompressor

\section{Introduction}

PoC (Push to Talk over Cellular) is the half-duplex voice service using VoIP technology based on $3 \mathrm{G}$ mobile cellular network, PoC can provide users with these functions, such as a single call, group call, group management, user classification, state real-time presence etc[1].

$\mathrm{PoC}$ is achieved through the VoIP technology, i.e. the form of transmission data packets is RTP/UDP/IPv4, the total packet headers are 40 bytes. If using GSM encoding, the voice payload is 33 bytes, the packet headers possess $55 \%$ of the entire length. Obviously, the effec- tive utilization of the link is very low, which is difficult to guarantee the QoS of the PoC. So 3GPP chose PDCP sublayer to solve the header compression[2].

The early header compression schemes were primarily designed for the wired link[3-4]. ROHC is designed against the wireless environment with the high delay and high error rate, the header compression scheme has the better robustness and the higher compression efficiency.

\section{PoC System Architecture}

The PoC network structure based on TDSCDMA network is shown in Fig.1, the server entities can directly be added in the GGSN of the UMTS core network[5].

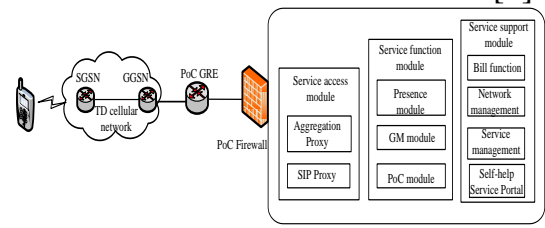

Fig.1 PoC system network architecture

The PoC client accesses the PoC service platform via the PS domain of TD network.

\section{Rohc Compression Principle}

The main reason why header compression can be done at all is the fact that there is significant redundancy between header fields, both within the same packet header but in particular between consecutive 
packets belonging to the same packet stream.

The ROHC compressor creates four profiles[6]: RTP, UDP, ESP and UNCOMPRESSED. The compression profiles establish an appropriate context for every stream belonging to themselves. Similarly, so does the decompressor. The relevant information from past packets is maintained in the context. The context information is used to compress and decompress subsequent packets. The compressor and decompressor constantly update their contexts upon certain events. Impairment events may lead to inconsistencies between the contexts of the compressor and decompressor, which in turn may cause incorrect decompression. So ROHC adopts CRC in the compressed packet, and utilizes the feedback information to ensure that the decompressor correctly updates the context in time.

\subsection{ROHC Operation States}

The compressor and the decompressor each has three states.

For compressor, the three states are: the Initialization and Refresh(IR), First Order(FO), and Second Order(SO) states.

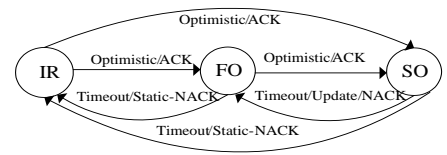

Fig.2 The compressor state transition

After some conditions are met, the above three states can transfer shown in Fig.2, but the compression is always started from the IR state.

The decompressor also exists in three states: No Context(NC) state; Static Context(SC) state; Full Context(FC) state. The state transitions among the three states are shown in Fig.3

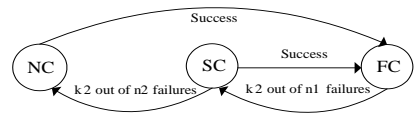

Fig.3 The decompressor State transition

\subsection{ROHC Operation Modes}

The ROHC scheme has three mode: Unidirectional(U-mode), Bidirectional Optimistic(O-mode), and Bidirectional Reliable(R-mode) mode. As is shown in Fig.4

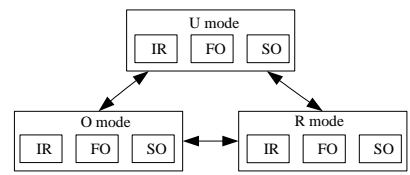

Fig.4 the relation of operation modes and states transition

The U-mode is without a feedback channel, in order to ensure the timely update of the context, the compressor need to periodically transfer to the low state; the O-mode and R-mode have the feedback channel.

\section{Rohc Implementation Process}

\subsection{Implementation of Compressor}

The operation state of the compressor will determine the compression efficiency. In the U-mode, to ensure the compression efficiency, improving the compression state and updating the context duly are very important; in the O-mode and Rmode, it is necessary to adjust the compression state according to the feedback to ensure the correctness of $\mathrm{ROHC}$ compressed packet chose.

(1) Implementation process of compressor

$\mathrm{PoC}$ is achieved via VoIP, so the main tasks of the compressor are:

(a) only compressing the received RTP/UDP/IP packets and generating the context and CID in order to ensure the decompressor can correctly decompress;

(b) to receive and process the feedback from the decompressor;

(c) completing the transfer of the three states, and optimizing the compression efficiency and robustness, as so on;

The specific process of the compressor is shown in Fig.5. 


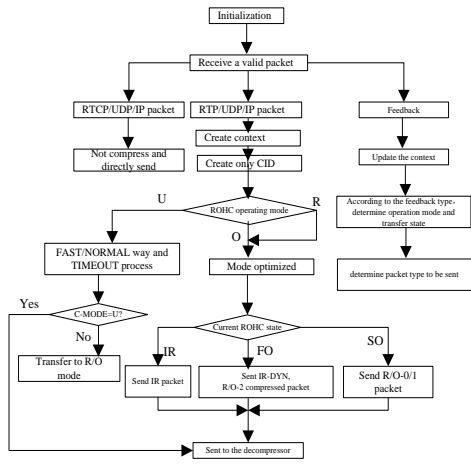

Fig.5 Implementation process of the compressor

(2) Implementation process of the feedback

The processing module of the feedback information in the compressor will deal with the feedback information from the decompressor, identify the type of the feedback, update the context of the compressor, and determine the mode conversion and the state transition according to the feedback, Fig.6 shows the operation process of the feedback.

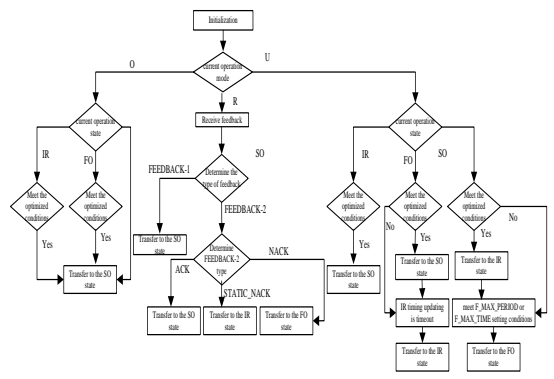

Fig.6 process of the feedback in compressor

\subsection{Implementation of Decompressor}

The decompressor re-generates the original data according to its own context and the W-LSB information in the receiving ROHC compressed packets, and will determine whether the generated data falls within a pre-set coding interval. If the generated data do not fall on the set coding interval, then move $\mathrm{N}$ coding intervals, if the data are not within the set coding interval, then discard the packet; If the data fall on the interval, the decompressor will restore the original RTP/UDP/IP packets and transfer them to the upper.

(1) Implementation process of decompressor

The main tasks of the decompressor are to decompress the ROHC packets, complete the conversion among the three modes and among the three decompression states, send the feedback to the compressor.

The process of the decompressor is shown in Fig. 7.

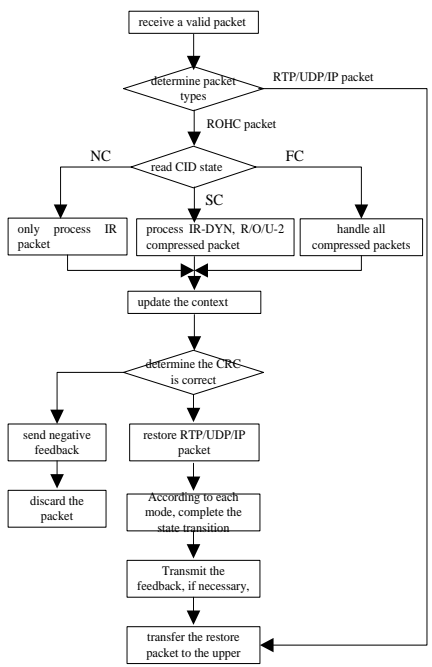

Fig.7 Implementation process of decompressor

When the decompressor receives the packet, the process is as follows:

Step 1: First, to distinguish the type of the data packet that has already received:

(a) if the type is RTCP/UDP/IP packets, without any treatment, directly transfer to the upper layer;

(b) if it is the feedback, which will be handed over to the compressor, and continue to process in the light of step c);

(c) if it is ROHC compressed packet, to determine its legitimacy and the type of ROHC compressed packet. 
Step 2: by reading the corresponding CID, to deal with the different packets in the different way.

Step 3: discard those that do not pass the CRC check, if the check passes, to decide whether it is need to update the context of the decompressor. Last, the correctly decompressed RTP/UDP/IP packets should be transmitted to the upper.

(2) improved algorithm of restoring compressed packets

The PoC is a real-time service, so the Feedback as little as possible, the best way is in the U-mode. The wireless link exists the possibility of a large number of packet loss and out-of-order, which will likely cause the compressor and decompressor with the inconsistent coding intervals to lead to incorrect decompression. The decompressor can only wait for U-mode to repair the context. This will affect the real-time seriously. Therefore, based on the traditional decompression context algorithm [7], the paper proposes an improved algorithm of the rapid reduction packet shown in Fig.8, the algorithm can repair the large number of packet loss in time.

\section{Conclusion}

The wireless network has the characteristics of higher error rate and longer transmission delay, which will guarantee the QoS for PoC difficultly. This article designs the implementation process of the corresponding modules according to the fundamentals of the compressor and the decompressor, in view of the strict delay requirements for $\mathrm{PoC}$, an improved algorithm suitable for the U-mode is brought forward, which can improve the robustness of the system and the utilization rate of the wireless link.

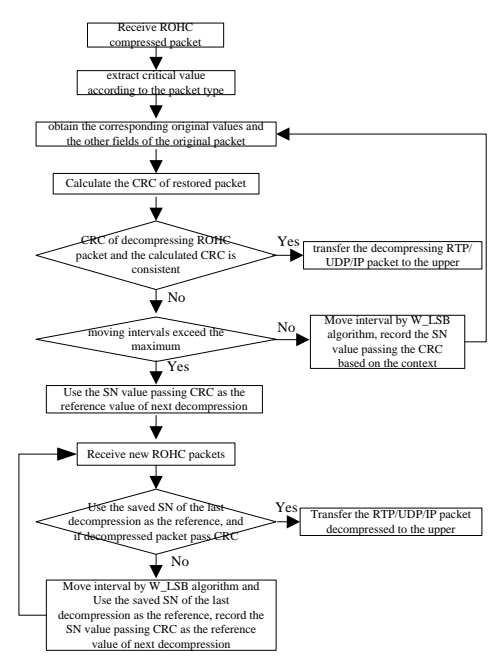

Fig.8 improved algorithm of restoring compressed packets

\section{References}

[1] OMA-RD-PoC-V1_0-20060609-A; Push to Talk over Cellular Requirements Approved Version 1.0[S]. 2006.

[2] 3GPP.TS 25.323 V8.3.0: Packet Data Convergence Protocol (PDCP) specification $[\mathrm{S}]$.

[3] IETF RFC 2508.Compressing IP/UDP/RTP heads for low-speed serial links[s].

[4] IETF RFC 2507. IP head compres$\operatorname{sion}[\mathrm{s}]$.

[5] Li Haiwen, Yu Gang, Huang Wei. Research of CPC for the Implementation of PoC Based on TD Network [J]. Television technology, 2012, 36(19).

[6] IETF RFC 3095.RObust Header Compression(ROHC) : Framework and four profiles: RTP, UDP, ESP and un-compressed[S]. 2001

[7] Zhang Fengxiang, Hong Peilin, Li Jinsheng. The windows based LSB codec correction algorithms used in ROHC mechanism [J]. Circuits and Systems, 2004,01 